\title{
The impact of the number of cores on tissue microarray studies investigating prostate cancer biomarkers
}

\author{
PIERRE TENNSTEDT $^{1}$, PATRICIA KÖSTER ${ }^{1}$, ANDREAS BRÜCHMANN ${ }^{1}$, MARTINA MIRLACHER $^{1}$, \\ ALEXANDER HAESE ${ }^{2}$, THOMAS STEUBER ${ }^{2}$, GUIDO SAUTER ${ }^{1}$, HARTWIG HULAND ${ }^{2}$, \\ MARKUS GRAEFEN $^{2}$, THORSTEN SCHLOMM ${ }^{2}$, SARAH MINNER ${ }^{1}$ and RONALD SIMON ${ }^{1}$ \\ ${ }^{1}$ Institute of Pathology, University Medical Center; ${ }^{2}$ Martini-Clinic, Prostate Cancer Center, \\ University Medical Center, Hamburg-Eppendorf, Germany
}

Received July 4, 2011; Accepted August 18, 2011

DOI: $10.3892 /$ ijo.2011.1216

\begin{abstract}
Most tissue microarray studies have used a single $0.6-\mathrm{mm}$ tissue core per donor tissue. It has been suggested that multiple cores per donor can increase the representativity of tissue microarray studies. To estimate the potential benefit of multiple cores, we analyzed Ki67 and p53 in triplet cores taken from three different areas of 3,261 prostate cancer tissue blocks. Both p53 and Ki67 labeling index were linked to advanced tumor stage ( $\mathrm{p}<0.0001$ each), Gleason score $(\mathrm{p}<0.0001)$, and early PSA recurrence $(\mathrm{p}<0.0001)$ independently of whether the 3 tissue spots were analyzed separately or combined for a consensus result. The rate of positive findings increased with the amount of analyzed tissue. The average Ki67 labeling index was higher in tumors with 3 interpretable spots $(5.3 \pm 5.6)$ as compared to two $(4.1 \pm 4.7)$ or one interpretable spot $(4.1 \pm 4.2, \mathrm{p}<0.0001)$. For p53, tumors with three interpretable spots were positive in $3.8 \%$ of cases, and tumors with 1 or 2 interpretable spots in $1.9 \%$ only $(\mathrm{p}=0.003)$. These data demonstrate that using multiple cores in a tissue microarray does not necessarily increase the ability to identify associations of biomarkers with tumor phenotype and prognosis but has always the disadvantage of additional work and tissue requirements. Multiple cores may even lead to statistical problems if unequal amounts of tissue are analyzed per tumor.
\end{abstract}

\section{Introduction}

Prostate cancer is the most frequent cancer in man and the second cause of cancer related death (1). Established pre-therapeutic

Correspondence to: Dr Ronald Simon, Institute of Pathology, University Medical Center Hamburg-Eppendorf, Martinistr. 52, D-20246 Hamburg, Germany

E-mail: r.simon@uke.uni-hamburg.de

Abbreviations: TMA, tissue microarray; BCR, biochemical tumor recurrence; LI, labeling index

Key words: prostate cancer, p53, Ki67 LI, TMA, protein, progression prognostic factors include Gleason score, tumor extension on biopsy, and serum PSA values (2). As many prostate cancer patients will not die from their disease and the spectrum of accepted treatment ranges from watchful waiting to radical surgery, a better prediction of the individual prognosis of prostate cancer patients would improve our ability for evidence based individual treatment recommendations. Unfortunately, studies investigating potential molecular prognostic factors are often too small for generating meaningful results. Most studies reporting potential prognostic biomarkers in prostate cancer describe findings in 40-100 tumors (3).

Tissue microarrays (TMAs) dramatically facilitate the in situ analysis of large numbers of cancer tissues (4). TMAs typically contain up to 600 tissue spots on one microscopic glass slide, with each arrayed tissue spot measuring $0.6 \mathrm{~mm}$. Our group and others have demonstrated the power of the TMA method to find associations between molecular features and clinical phenotype and prognosis. In these studies, we have reproduced virtually all established biomarker associations in a TMA format with a single tissue core per tumor $(3,5-8)$. In prostate cancer studies we also used TMAs containing just one $0.6 \mathrm{~mm}$ spot per tumor to demonstrate a prognostic role of numerous markers including p53 (9), CD10 (10), mast cell count (11), EGFR (12), HER2 (13), Ki67 labeling index (Ki67 LI) (14), PSA (15), or copy number changes involving chromosomes $8 \mathrm{p} / 8 \mathrm{q}(16)$.

Some authors have reported that the analysis of multiple spots per donor tissue can further improve the results of TMA studies (17-24). Especially in prostate cancer, it has been suggested that 3-4 spots should be used per tumor (25). Analysis of 4 spots adds substantial workload to a TMA study since it involves the use of 4 times more tissue and work time both for TMA making and analysis. In order to justify this additional work, it would be important to demonstrate that the higher number of analyzed tissue spots leads to better associations with important parameters such as patient outcome or tumor phenotype. To generate more data on the infrastructural needs for prostate cancer TMA studies, we analyzed two potentially heterogeneous biomarkers in three different spots of a prostate cancer TMA and compared the data with clinical outcome in 2,385 patients. 


\section{Patients and methods}

Patients/tissue microarrays. A previously manufactured tissue microarray (TMA) was available for this project. The TMA was manufactured from radical prostatectomy specimens from 3,261 patients, treated at the Department of Urology, University Medical Center Hamburg-Eppendorf between 1992 and 2005. Patients were selected having clinically organ confined (early stage) tumors. Follow-up data were available for 2,385 patients, ranging from 1 to 144 months (mean 34.9 months). The clinicopathological features of the arrayed prostate cancers are given in Table I. All prostatectomy specimens were analyzed according to a standard procedure. All prostates were completely paraffinembedded, including whole-mount sections as previously described (26). All hematoxylin and eosin (H\&E) stained histological sections from all prostatectomy specimens were reviewed for the purpose of this study and representative tumor areas were marked on the slides. Three sets of the TMA, each set consisting of 7 TMA blocks, were manufactured. For each TMA block, one 0.6-mm tissue core was punched out from the index tumors of each case, and transferred in a tissue microarray (TMA) format as described (27). For each of the three TMA sets, the 3,261 cores were distributed among the 7 TMA blocks each containing 129-522 tumor samples. Each TMA block also contained various control tissues including normal prostate tissue, other normal tissues and a set of tumor tissues including several colon and breast cancers.

Immunohistochemistry. Freshly cut TMA sections were used for all immunostainings. Ki67 staining was performed as described before (13). In brief, the Mibl antibody (1:400, Dianova) was used for Ki67 protein detection after steaming the sections at $98^{\circ} \mathrm{C}$ for $20 \mathrm{~min}$ in citrate buffer $\mathrm{pH}$ 9.0. P53 immunohistochemistry was performed as described before (9). In brief, TMA sections were pretreated in a microwave oven in $\mathrm{pH} 7.8$ buffer. The primary antibody (clone DO1, Oncogene) was diluted 1:3,600 for immunostaining. The Envision system (Dako) was used for both antibodies to visualize the immunostainings. Tumors with known positivity were used as positive controls. The primary antibody was omitted for negative controls. Nuclei were considered Ki67 positive if any nuclear staining was seen. The Ki67 LI (percentage of Ki67 positive cells) was determined by counting the fraction of positive tumor cell nuclei at intervals of $\leq 5, \leq 10$, $\leq 20, \leq 30 \%$ etc. in 100 tumor cells in the hot spot area of each arrayed tissue sample. If $<100$ cells were present in a TMA spot, all tumor cells were counted. $\mathrm{P} 53$ positivity was assumed if $>1 \%$ of tumor cells showed unequivocal nuclear staining.

All slides from the three TMA sets were immunostained for low molecular weight cytokeratins to assure presence of cancer cells in the TMA spots. For this purpose, the antibody $34 \beta E 12$ (clone MA903; Dako; 1:12,5) was used for basal cell detection after boiling the sections in an autoclave in citrate buffer, $\mathrm{pH}$ 7.8.

Analysis of the three TMA sets for Ki67 and p53 immunostaining resulted in data points from three spots per tumor/ patient. Data on the first TMA set had previously been published $(3,9)$. The second and third set of TMAs were stained together using the same protocols as previously published.

Statistical analyses. Statistical calculations were performed with JPM 8.01 software (SAS Institute Inc., NC, USA). Contingency
Table I. Clinico-pathological features of the entire study cohort of 3,261 patients. ${ }^{a}$

\begin{tabular}{|c|c|c|}
\hline Characteristic & $\begin{array}{c}\text { No. on TMA } \\
n=3,261\end{array}$ & $\begin{array}{l}\text { No. (\%) with clinic } \\
\text { follow-up } \\
n=2,385\end{array}$ \\
\hline \multicolumn{3}{|c|}{ Follow-up (months) } \\
\hline Mean & & 34.9 \\
\hline Median & & 30.5 \\
\hline PSA recurrence & & $526 \quad(22.1)$ \\
\hline \multicolumn{3}{|l|}{ Age (years) } \\
\hline$<50$ & 83 & $65(78.3)$ \\
\hline $50-60$ & 998 & $759 \quad(76.1)$ \\
\hline $60-70$ & 1,807 & $1,376(76.1)$ \\
\hline$>70$ & 175 & 155 \\
\hline
\end{tabular}

Pre-treatment PSA

$(\mathrm{ng} / \mathrm{ml})$

$<4$

513

$371(72.3)$

4-10

1,673

$1,244(74.4)$

10-20

641

$522 \quad(81.4)$

$>20$

225

$201 \quad(89.3)$

pT category

(AJCC 2002)

pT2

2,080

$1,500(72.1)$

pT3a

609

$519 \quad(85.2)$

pT3b

372

322 (86.6)

pT4

42

$42(100.0)$

$\begin{array}{lrrr}\text { Gleason score } & & & \\ \leq 3+3 & 1,426 & 1,015 & (71.2) \\ 3+4 & 1,311 & 1,050 & (80.1) \\ 4+3 & 313 & 274 & (87.5) \\ \geq 4+4 & 55 & 46 & (83.6)\end{array}$

$\begin{array}{lrrr}\text { pN category } & & & \\ \text { pN0 } & 1,544 & 1,425 & (92.3) \\ \text { pN1 } & 96 & 88 & (91.7) \\ \text { pNx } & 1,457 & 866 & (59.4)\end{array}$

Surgical margin

$\begin{array}{lrrr}\text { Negative } & 2,475 & 1,858 & (75.1) \\ \text { Positive } & 627 & 525 & (83.7)\end{array}$

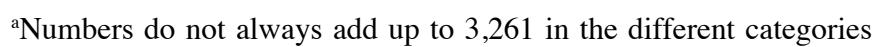
because of cases with missing data. AJCC, American Joint Committee on Cancer.

tables were calculated and the $\chi^{2}$ test (likelihood) was used to see significant associations. For continuous scaled variables 
Table II. Association between the amount of analyzed tissue, Ki67 LI \pm SD.

\begin{tabular}{|c|c|c|c|c|c|c|}
\hline \multirow[b]{2}{*}{$\begin{array}{l}\text { Interpretable } \\
\text { spots }(0.6 \mathrm{~mm})\end{array}$} & \multirow[b]{2}{*}{$\begin{array}{l}\text { Analyzed tissue } \\
\text { area }\left(\mathrm{mm}^{2}\right)\end{array}$} & \multicolumn{3}{|c|}{ Ki67 } & \multicolumn{2}{|r|}{ p53 } \\
\hline & & $\mathrm{n}$ & Average LI & Highest LI & $\mathrm{n}$ & $\%$ positive \\
\hline One & 1.13 & 731 & $4.1 \pm 4.2$ & $4.1 \pm 4.2$ & 725 & 2.3 \\
\hline Two & 2.26 & 489 & $4.1 \pm 4.7$ & $5.3 \pm 5.6$ & 476 & 1.3 \\
\hline Three & 3.39 & 1637 & $5.3 \pm 5.6$ & $7.7 \pm 7.3$ & 1668 & 3.8 \\
\hline p-value & & & $<0.0001$ & $<0.0001$ & & $\begin{array}{l}0.0048 \\
(0.003)^{\mathrm{a}}\end{array}$ \\
\hline
\end{tabular}

Analyzable spots for Ki67 and p53. ${ }^{\text {a }}$-value if p53-positive tumors with 1 or 2 analyzable spots are combined.

the ANOVA test was performed and standard deviation was indicated. Survival curves were calculated by the Kaplan-Meier method and compared with the log-rank test.

\section{Results}

Technical issues. Unequivocal prostate cancer was present in 2,525, 2,075 and 2,197 of 3,261 arrayed tissue samples from our three prostate cancer prognosis TMA sets (TMA1-3), respectively. Non-informative cases were caused by missing spots in the TMAs (TMA1 1.9\%, TMA2 1.4\%, TMA3 2.1\%) or absence of unequivocal invasive cancer tissue in the associated $34 \beta \mathrm{E} 12$ IHC (TMA1 20.5\%, TMA2 35.0\%, and TMA3 30.5\%). The latter spots either contained normal prostatic tissue, high-grade prostatic intraepithelial neoplasia (PIN), or stromal tissue only.

Ki67 LI immunohistochemistry. Ki67 was evaluable in one of the three spots in 731 cases, in 2 spots in 489 cases, and in all 3 spots in 1,637 cases. The Ki67 LI was significantly linked to the amount of analyzed tissue: cancers with three analyzable spots had an average Ki67 LI of 5.3 \pm 5.6 whereas tumors with only one interpretable tissue spot averaged a Ki67 LI of 4.1 \pm 4.2 ( $\mathrm{p}<0.0001$; Table II). This association was even more evident if only the spot with the highest Ki67 LI was considered in cases where more than one spot was analyzable $(p<0.0001$; Table II). In order to avoid a bias because of the different amount of analyzed tissues per tumor, the further analysis was restricted to the subset of 1,637 cancers where all three spots were analyzable. When the three TMA sets were analyzed separately, the Ki67 LI was significantly linked to non-organ-confined tumors $(\mathrm{p}<0.0001)$ and higher Gleason score $(\mathrm{p}<0.0001$; Table III) in all three TMA sets. No improvement of the results was observed if the results of the 3 TMA sets where jointly analyzed, classifying a tumor as positive if at least one of the three spots was scored positive, neither for the average Ki67, nor if only the spot with the highest Ki67 was considered (Table III). Representative images of Ki67 stained tissue spots are shown in Fig. la and b.

For all three TMA sets, presence of Ki67 staining was significantly related to biochemical tumor recurrence (BCR), as defined by a persisting or rising postoperative PSA $(>0.1 \mathrm{ng} /$ $\mathrm{ml})$. This was true if quartiles of 25,50 , and $75 \%$ were used
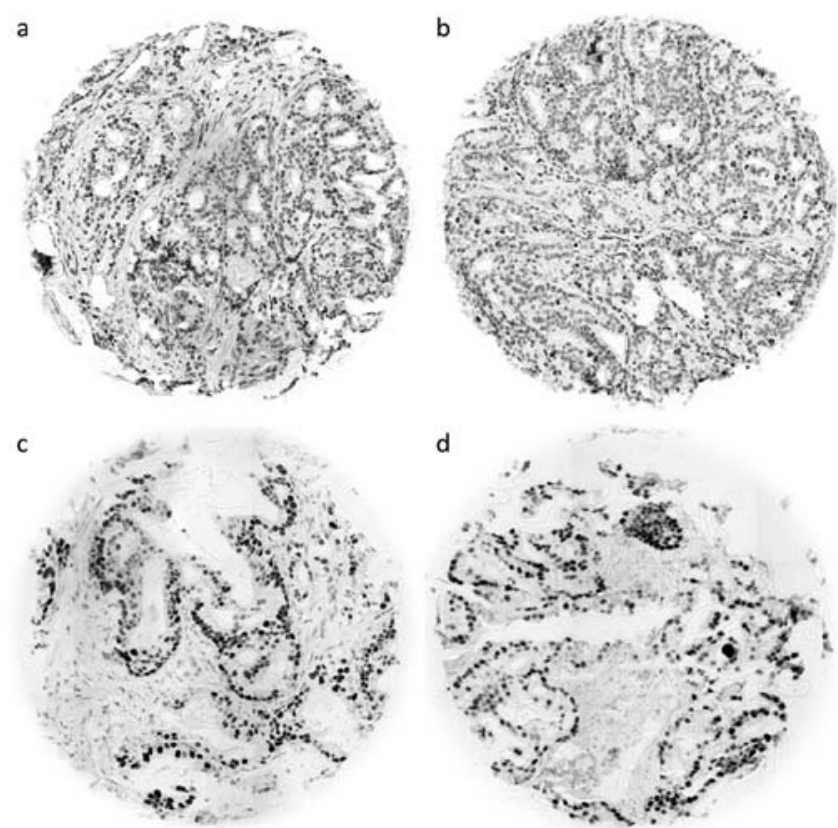

Figure 1. Examples of Ki67 (a and b) and p53 (c and d) immunostaining in two spots each obtained from the same tumor.

to defined Ki67 LI levels in the three TMAs (Fig. 2a-c), or if thresholds for Ki67 LI levels were arbitrarily selected (no staining, $<10,10-20$ and $\geq 20 \%$; $<<0.0001$ each, Fig. 2f-g). Also the combination of the results from the three TMAs did not lead to an improvement as compared to the separate analysis of the TMA sets alone, neither for the average Ki67 LI (Fig. 2d and e; $p<0.0001$ each), nor if only the highest Ki67 LI of the three spots was rated (Fig. $2 \mathrm{i}$ and j; p<0.0001).

P53 immunohistochemistry. In all 3 TMA sets, one spot was evaluable in 725 cases, 2 spots in 476 cases, and 3 spots in 1,668 cases. Comparable to the Ki67 findings, also the fraction of p53-positive cancers was linked to the amount of analyzed tissue. Despite the overall small fraction of p53-positive tumors (2.5\%), cancers with 3 analyzable spots were significantly more positive for p53 $(3.8 \%)$ than cancers with 1 or 2 spots (combined $1.9 \%$, $\mathrm{p}=0.003)$. All data are summarized in Table II. Also for $\mathrm{p} 53$ 
Table III. Ki67 LI ( \pm SD) according to tumor stage, Gleason score, and PSA level in all three TMA sets and a combined analysis for average and highest Ki67 LI.

\begin{tabular}{|c|c|c|c|c|c|c|}
\hline Parameter & $\begin{array}{c}\text { TMA 1 } \\
(\mathrm{n}=1,637)\end{array}$ & $\begin{array}{c}\text { TMA 2 } \\
(\mathrm{n}=1,637)\end{array}$ & $\begin{array}{c}\text { TMA 3 } \\
(\mathrm{n}=1,637)\end{array}$ & $\begin{array}{c}\text { Average } \\
(\mathrm{n}=1,637)\end{array}$ & $\begin{array}{l}\text { Highest } \\
(\mathrm{n}=1,637)\end{array}$ & p-value \\
\hline \multicolumn{7}{|c|}{ Tumor stage } \\
\hline 2 & $4.10 \pm 4.1$ & $4.93 \pm 5.6$ & $4.81 \pm 5.7$ & $4.61 \pm 4.6$ & $6.74 \pm 6.1$ & $0.0009^{b}$ \\
\hline $3 a$ & $5.54 \pm 5.9$ & $6.50 \pm 7.1$ & $6.51 \pm 7.5$ & $6.18 \pm 6.2$ & $8.87 \pm 7.9$ & 0.0999 \\
\hline $3 b$ & $5.92 \pm 6.1$ & $6.94 \pm 7.4$ & $6.76 \pm 7.6$ & $6.54 \pm 6.3$ & $9.48 \pm 8.3$ & 0.2943 \\
\hline 4 & $9.82 \pm 16.3$ & $9.78 \pm 14.2$ & $10.22 \pm 15.8$ & $9.94 \pm 15.0$ & $12.93 \pm 17.1$ & 0.9933 \\
\hline p-value & $<0.0001$ & $<0.0001$ & $<0.0001$ & $<0.0001$ & $<0.0001$ & \\
\hline \multicolumn{7}{|c|}{ Gleason score } \\
\hline$\leq 3+3$ & $3.75 \pm 3.9$ & $4.33 \pm 5.1$ & $4.17 \pm 4.9$ & $4.10 \pm 4.1$ & $6.02 \pm 5.5$ & 0.0692 \\
\hline $3+4$ & $4.70 \pm 4.5$ & $5.72 \pm 6.0$ & $5.74 \pm 6.3$ & $5.40 \pm 4.9$ & $7.84 \pm 6.6$ & $0.0005^{\mathrm{b}}$ \\
\hline $4+3$ & $7.19 \pm 7.3$ & $8.38 \pm 8.7$ & $8.32 \pm 9.4$ & $8.0 \pm 7.7$ & $11.32 \pm 9.6$ & 0.2944 \\
\hline$\geq 4+4$ & $11.15 \pm 15.7$ & $12.34 \pm 13.8$ & $11.17 \pm 14.7$ & $11.6 \pm 14.1$ & $15.17 \pm 16.3$ & 0.9278 \\
\hline p-value & $<0.0001$ & $<0.0001$ & $<0.0001$ & $<0.0001$ & $<0.0001$ & \\
\hline \multicolumn{7}{|l|}{ PSA level } \\
\hline$<4$ & $5.02 \pm 6.5$ & $5.87 \pm 7.1$ & $5.82 \pm 7.5$ & $5.57 \pm 6.5$ & $8.00 \pm 8.0$ & 0.3598 \\
\hline $4-10$ & $4.73 \pm 4.9$ & $5.61 \pm 6.3$ & $5.54 \pm 6.4$ & $5.29 \pm 5.2$ & $7.66 \pm 7.0$ & $0.0051^{\mathrm{b}}$ \\
\hline $10-20$ & $4.92 \pm 5.8$ & $5.83 \pm 6.7$ & $5.66 \pm 7.1$ & $5.47 \pm 5.9$ & $7.90 \pm 7.6$ & 0.1361 \\
\hline$>20$ & $4.44 \pm 5.2$ & $5.20 \pm 6.7$ & $4.96 \pm 6.5$ & $4.86 \pm 5.6$ & $7.14 \pm 7.1$ & 0.5473 \\
\hline p-value & 0.7227 & 0.7387 & 0.6563 & 0.6427 & 0.6779 & \\
\hline
\end{tabular}

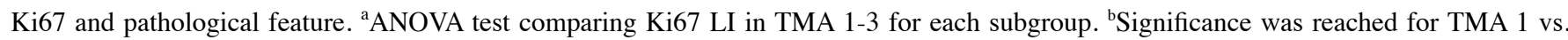
TMA 2 and TMA 1 vs. TMA 3.

Table IV. p53 expression (\% positive cases) according to tumor stage, Gleason score, and PSA level, in all three TMA sets and a combined analysis.

\begin{tabular}{|c|c|c|c|c|c|}
\hline Parameter & $\begin{array}{c}\text { TMA 1 } \\
(\mathrm{n}=1,668)\end{array}$ & $\begin{array}{c}\text { TMA 2 } \\
(\mathrm{n}=1,668)\end{array}$ & $\begin{array}{c}\text { TMA } 3 \\
(n=1,668)\end{array}$ & $\begin{array}{l}\text { Combined } \\
(\mathrm{n}=1,668)\end{array}$ & p-value ${ }^{a}$ \\
\hline \multicolumn{6}{|c|}{ Tumor stage } \\
\hline 2 & 1.02 & 1.84 & 1.43 & 2.24 & 0.3087 \\
\hline $3 a$ & 3.32 & 4.43 & 4.71 & 4.99 & 0.6048 \\
\hline $3 b$ & 6.73 & 5.77 & 5.77 & 8.17 & 0.8955 \\
\hline 4 & 11.11 & 11.11 & 11.11 & 11.11 & 1.0 \\
\hline p-value & $<0.0001$ & $<0.0001$ & $<0.0001$ & 0.0002 & \\
\hline \multicolumn{6}{|c|}{ Gleason core } \\
\hline$\leq 3+3$ & 0.92 & 1.53 & 1.23 & 1.84 & 0.59963 \\
\hline+4 & 2.17 & 2.60 & 2.32 & 3.18 & 0.86494 \\
\hline+3 & 7.61 & 8.12 & 8.63 & 10.15 & 0.9342 \\
\hline$\geq 4+4$ & 8.57 & 14.29 & 14.29 & 17.14 & 0.6900 \\
\hline p-value & $<0.0001$ & $<0.0001$ & $<0.0001$ & $<0.0001$ & \\
\hline \multicolumn{6}{|l|}{ PSA level } \\
\hline$<4$ & 3.20 & 3.65 & 3.2 & 4.11 & 0.9547 \\
\hline $4-10$ & 1.61 & 2.61 & 2.36 & 3.23 & 0.3511 \\
\hline $10-20$ & 3.73 & 3.73 & 3.73 & 5.07 & 1.0 \\
\hline$>20$ & 2.67 & 3.33 & 3.33 & 3.33 & 0.9272 \\
\hline p-value & 0.1452 & 0.7076 & 0.5936 & 0.4943 & \\
\hline
\end{tabular}

p53 and pathological feature. ${ }^{a} \chi^{2}$ test comparing p53 positivity in TMA 1-3 for each subgroup. 

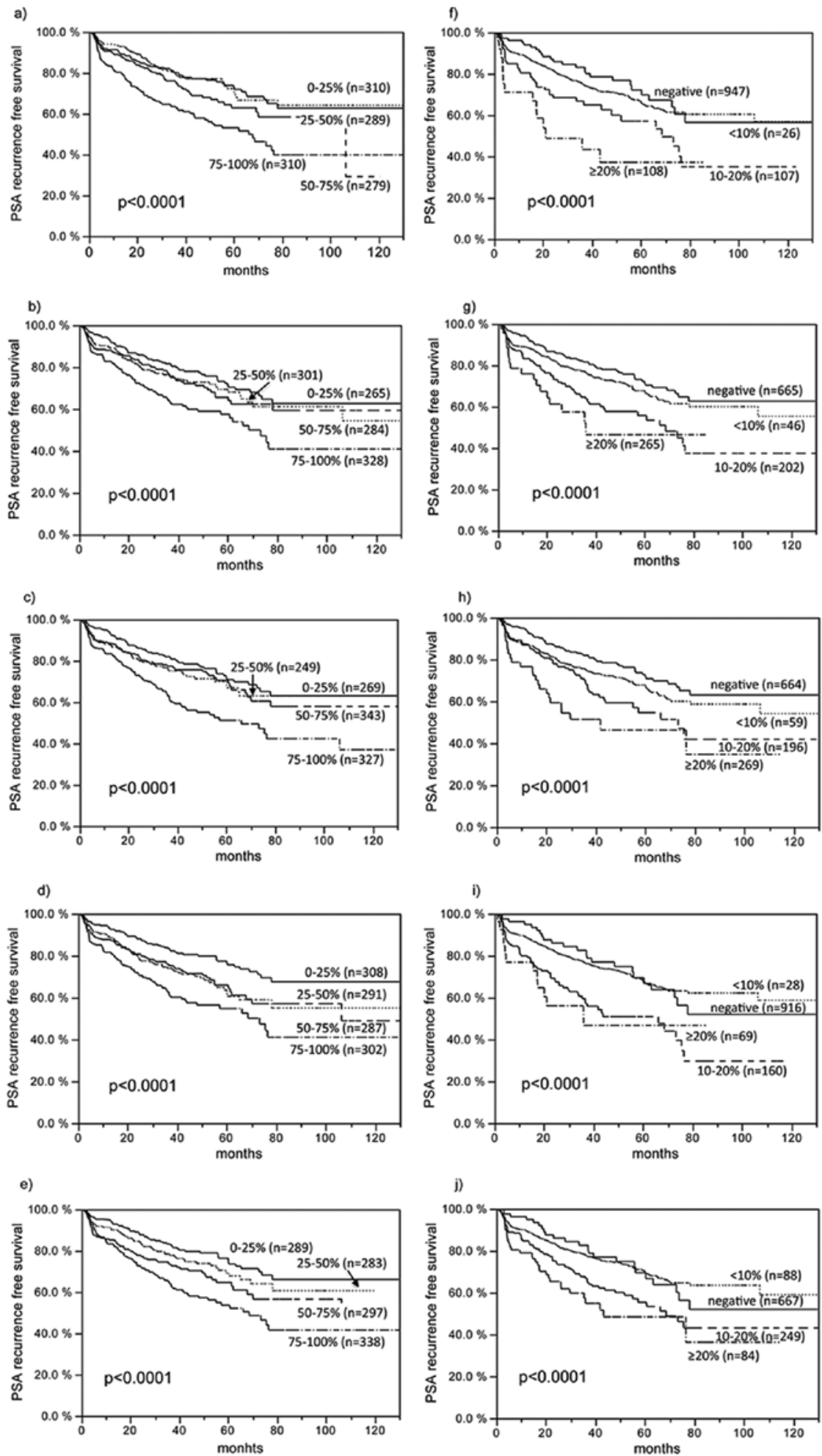

Figure 2. Kaplan-Meier analysis of the association between biochemical recurrence and the Ki67 LI. Left hand panels, tumors were subdivided in 4 expression groups according to the 25, 50, and 75\% quartiles. (a-c) Separate analysis of: (a) TMA set no. 1; (b) TMA set no. 2; and (c) TMA set no. 3; and (d and e) combined analysis of the three TMA sets if the: (d) average Ki67 LI; or (e) highest Ki67 LI of the three spots was scored. Right hand panels, tumors were subdivided in 4 expression groups reflecting no detectable Ki67 staining, $<10 \%, 10-20 \%$, and $\geq 20 \%$ Ki67 LI. (f-h) Separate analysis of: (f) TMA set no. 1; (g) TMA set no. 2; and (h) TMA set no. 3, and (i and j) combined analysis of the three TMA sets if the: (i) average Ki67 LI; or (j) highest Ki67 LI of the three spots was scored.

evaluation, analysis was restricted to the subset of cases where all three spots of each TMA were analyzable. When comparing p53 positivity in TMA 1-3 in subgroup analysis by tumor stage, Gleason score, and PSA level no significant differences were found. All results are summarized in Table IV. Representative images of p53 stained tissue spots are shown in Fig. 1c and d. P53 positivity was significantly increased with non-organ-confined tumors ( $<<0.0001$ in each TMA set) and higher Gleason score $(p<0.0001$ in each TMA set). For all 3 analyzed TMA sets, p53 positivity was significantly related to PSA recurrence $(\mathrm{p}<0.0001$, 

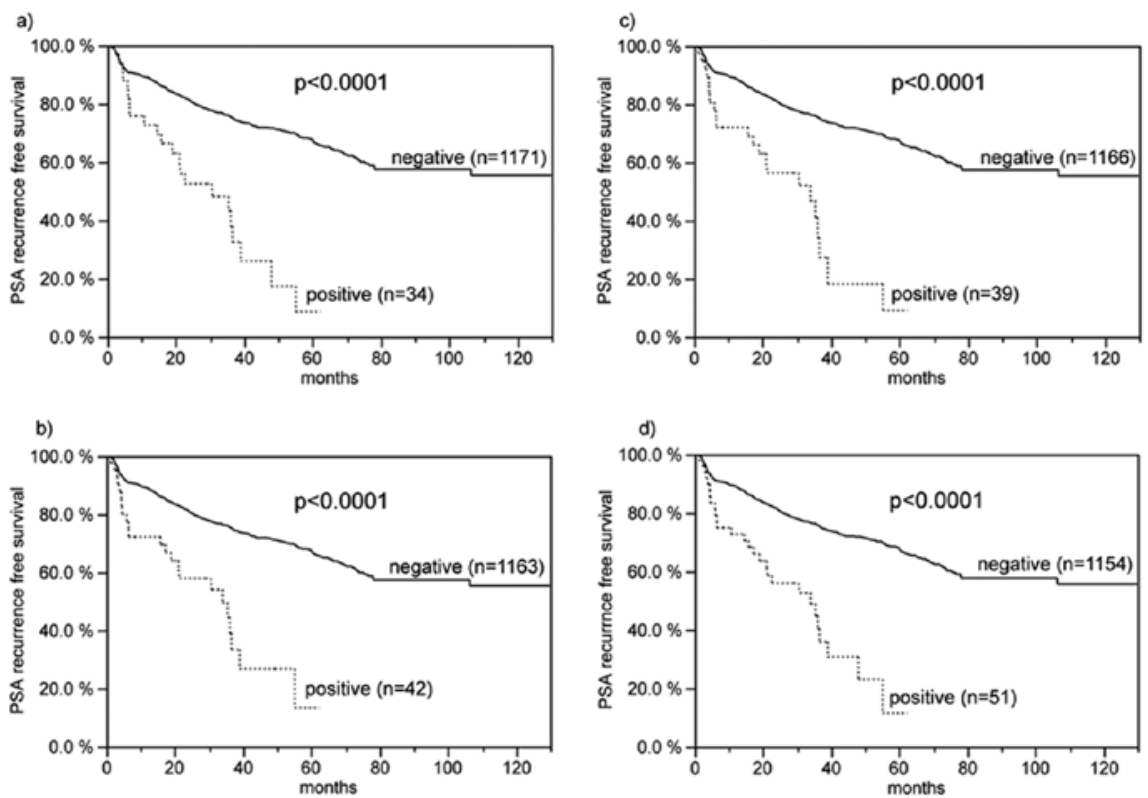

Figure 3. Kaplan-Meier analysis of the association between biochemical recurrence and immunohistochemical p53 positivity. Separate analysis of: (a) TMA set no. 1; (b) TMA set no. 2; and (c) TMA set no. 3; and (d) combined analysis of the three TMA sets. For the combined analysis, a tumor was rated p53-positive if at least one of the three TMA spots showed a positive result.

Fig. 3a-c). The combination of all three TMAs classifying a tumor as positive if at least one spot was scored positive did not lead to a clear improvement as compared to the separate analyses of the TMA sets (Fig. 3d; $p<0.0001$ ).

\section{Discussion}

The main purpose of this study was to determine whether the use of three different tissue cores per tumor generally offers an advantage to prostate cancer TMA studies that justifies the substantial logistic and economical extra burden. The data show that the prognostic relevance for $\mathrm{p} 53$ and Ki67 could be clearly identified in each of three different TMAs. The combination of the results from three different TMAs did not discernable improve the statistical significance of our results. The results of this study support the use of TMAs containing one $0.6 \mathrm{~mm}$ spot per tumor for biomarker evaluation in prostate cancer.

Other authors have come to different conclusions based on studies comparing immunohistochemistry results obtained on large sections and on corresponding TMAs composed of variable numbers of spots per tumor. Several such studies have shown that the number of positive cases increased with the numbers of TMA spots analyzed in different cancer types $(17,18,22)$. For example, Kristiansen et al (24) used duplicate prostate cancer cores and reported that in some cases only one core showed at least some immunoreactivity while the other core was completely AMACR-negative. Camp et al (17) reported that analysis of one core matched the staining pattern of the whole section in more than $90 \%$ of samples, but analysis of 2 cores in more than 95\% for HER2, PR and ER in breast cancer. Similarly, Hoos et al (18) found that 3 or 2 cores matched the staining patterns of Ki67 and pRB in the whole section in 94 and $96 \%$, but 1 core in only 89 and $91 \%$ of samples. In prostate cancer, Rubin et al (25) found that analysis of 3 spots best reflected the Ki67 LI in standard pathology immunohistochemistry slides.

Based on such findings, analyzing three or more spots was strongly recommended for the search of clinically relevant biomarkers in prostate cancer by several authors $(25,28)$. In our opinion, this proposal is based on two disputable assumptions. Assumption no. 1 is that the analysis of one large section from a radical prostatectomy specimen is the 'gold standard' for finding clinically relevant biomarkers for prostate cancer. We believe that this assumption is not supported by evidence considering that large sections represent only a tiny amount of a prostate cancer. For example, in a series of 1,657 prostatectomy specimens diagnosed in our laboratory in 2010, the average tumor volume was $4.4 \mathrm{~cm}^{3}$. If a large section contains $2 \times 1 \mathrm{~cm}$ of cancer, this $4-\mu \mathrm{m}$ section only analyzes $0.00008 \mathrm{~cm}^{3}$ of tumor, which equals $0.0018 \%$ of the entire tumor. It has never been shown that this amount of tissue really represents the molecular diversity of a prostate cancer. It is also intuitive for genitourinary pathologists that relevant prostate cancer heterogeneity - such as tertiary Gleason score - is not necessarily found on every unselected slide of a cancer patient. Moreover, clinically relevant biomarkers must be detectable on smaller tissue samples, e.g., prostate biopsies because treatment decisions must be made before prostatectomy specimens are available for molecular analyses. Assumption no. 2 is that the higher rate of positive cases introduced by the analysis of multiple cores always reflects expression heterogeneity. However, immunohistochemical stainings can lead to staining artifacts, for which the likelihood also increases if more tissue is subjected to immunostaining. It is therefore possible that the higher positivity rate in TMAs with multiple spots per tumor or on large section is partly due to a higher rate of false positivity through non-specific staining.

To determine, how many tissue spots are needed for investigating molecular markers on TMAs it appears to be optimal 
to compare both TMA and large section data with clinical parameters rather than comparing one with each other. This labor-intensive approach was pursued in one study analyzing more than 600 breast cancers on large sections and on four different TMA spots for $\mathrm{p} 53$, estrogen receptor and progesterone receptor (6). While largely identical results were found on large sections and all 'single spot TMAs' for ER and PR, the results were more diverse for $\mathrm{p} 53$. In that study, all individual TMAs showed p53 positivity rates between 15 and $21 \%$ and the positive TMA results were always strongly linked to poor prognosis. In the same study, the large sections showed markedly higher positivity rates $(43 \%)$ but no significant association was seen with prognosis. Importantly, these breast cancers with positivity on large sections but not on TMAs had a prognosis identical to the prognosis of tumors that were p53 negative on large sections (4). At least in the study by Torhorst et al (6), it became evident, that the higher rate of positive cases introduced by the analysis of more tissue was not an advantage justifying the very substantial extra work. It was concluded that either staining artifacts or (less likely) lack of biological significance of small focal p53 staining, that could not be detected on a TMA containing one $0.6 \mathrm{~mm}$ spot per tumor, was driving the higher positivity rate in large sections. Similar to the study by Torhorst et al, Rubin et al (25) studied the effect of Ki67 expression on patient prognosis in a prostate cancer tissue microarray containing 10 cores per prostate cancer tissue block. Corroborating the findings of Torhorst et al (6) and of our present study, analysis of one to two cores was sufficient to find the known prognostic relevance of Ki67 LI even in multivariate analysis. A refined analysis suggested that 3-4 cores could give better results, but we attribute this observation to the relatively small size of this patient set $(n=88)$.

The use of multiple samples per tumor on a TMA has multiple disadvantages. Most obviously, it leads to a sensible multiplication of tissue requirements, work time and costs. Moreover, our data demonstrate, that a statistical bias can be introduced by using multiple cores per tumor. In our study, the highest Ki67 LI decreased from $7.7 \pm 7.3$ in 1,637 cancers with three interpretable spots to $4.1 \pm 4.2$ in 731 cancers with two interpretable spot only. The same association was also seen for $\mathrm{p} 53$, despite the overall small number of p53-positive cases. Of 1,668 tumors 3.8\% with three interpretable spots, but only $1.9 \%$ of 1,201 tumors with 1 or 2 interpretable spots showed p53 immunostaining. These data demonstrate, how the likelihood of an overall positive result will be higher in cancers with more interpretable spots than in cancers with only one or a few interpretable spots. All studies analyzing the impact of multiple samples per cancer have also shown that the fraction of positive cases increases with the number of analyzed spots. Unfortunately, arraying of 3 spots per tumor will not automatically lead to 3 interpretable samples per tumor. In our opinion, the same line of arguments supports the use of as small as possible tissue spots per tumor. In small $(0.6 \mathrm{~mm})$ tissue spots, tumor cells are often seen either in all, or in none, of the analyzed tissue, resulting in maximal standardization of the amount of tissue analyzed per tumor. The larger the arrayed spot or the arrayed amount of tissue, the higher will be the variability of the amount of cancer tissue analyzed per patient.

With respect to the individual biomarkers analyzed in this study, a few more conclusions can be drawn. For Ki67, the data support the prognostic relevance of tumor cell proliferation (14) and that the proliferative activity even has importance if small biopsy samples are analyzed (29). Given the heterogeneity of tumor cell proliferation within cancers, it has earlier been discussed whether proliferation hot spots or randomly selected areas better reflect tumor proliferation properties $(14,30)$. Despite the punched tumor areas in our TMA sets had not been selected for Ki67 expression hotspots, we found the prognostic relevance in all three individual tissue spots, arguing against a general superiority of hot spots as compared to analysis of random fields.

In conclusion, these data confirm that prognostic markers can be evaluated in studies using large prostate cancer TMAs containing one spot per cancer. Accordingly, we have successfully used our prostate cancer prognosis TMA to show a significant impact of EGFR (12), HER2 (13), p53 (9), Ki67 (3), PSMA (31), CD10 (10), CD117 (11), ANXA3 (32), copy number alteration of chromosomes 8p and 8q (16), and microvessel density (33) on early PSA recurrence in earlier studies. The analysis of two different markers, one of them Ki67 LI, which is notorious for being heterogeneous in cancers, did not provide any evidence for advantages provided by the use of multiple cores per tumor. Considering the additional work as well as possible statistical bias introduced by variable numbers of interpretable tissue spots per tumor, the use of just one spot per tumor TMAs is suggested.

\section{Acknowledgements}

This study was supported by the German Federal Ministry of Education and Science in the framework of the program for medical genome research (FKZ:01GS08189). The authors declare no conflict of interest.

\section{References}

1. Jemal A, Siegel R, Ward E, Hao Y, Xu J and Thun MJ: Cancer Statistics, 2009. CA Cancer J Clin 59: 225-249, 2009.

2. Eble JN, Sauter G,Epstein JI and Sesterhenn IA (eds.): World Health Organization Classification of Tumors. Pathology and Genetics of Tumors of the Urinary System and Male Genital Organs. IARC Press, Lyon, 2004.

3. Schlomm T, Erbersdobler A, Mirlacher M and Sauter G: Molecular staging of prostate cancer in the year 2007. World J Urol 25: 19-30, 2007.

4. Sauter G, Simon R and Hillan K: Tissue microarrays in drug discovery. Nat Rev Drug Discov 2: 962-972, 2003.

5. Simon R, Atefy R, Wagner U, et al: HER-2 and TOP2A coamplification in urinary bladder cancer. Int J Cancer 107: 764-772, 2003.

6. Torhorst J, Bucher C, Kononen J, et al: Tissue microarrays for rapid linking of molecular changes to clinical endpoints. Am J Pathol 159: 2249-2256, 2001.

7. Al-Kuraya K, Schraml P, Sheikh S, et al: Predominance of high-grade pathway in breast cancer development of Middle East women. Mod Pathol 18: 891-897, 2005.

8. Al-Kuraya K, Schraml P, Torhorst J, et al: Prognostic relevance of gene amplifications and coamplifications in breast cancer. Cancer Res 64: 8534-8540, 2004.

9. Schlomm T, Iwers L, Kirstein P, et al: Clinical significance of p53 alterations in surgically treated prostate cancers. Mod Pathol 21: 1371-1379, 2008.

10. Fleischmann A, Schlomm T, Huland H, et al: Distinct subcellular expression patterns of neutral endopeptidase (CD10) in prostate cancer predict diverging clinical courses in surgically treated patients. Clin Cancer Res 14: 7838-7842, 2008.

11. Fleischmann A, Schlomm T, Kollermann J, et al: Immunological microenvironment in prostate cancer: high mast cell densities are associated with favorable tumor characteristics and good prognosis. Prostate 69: 976-981, 2009. 
12. Schlomm T, Kirstein P, Iwers L, et al: Clinical significance of epidermal growth factor receptor protein overexpression and gene copy number gains in prostate cancer. Clin Cancer Res 13: 6579-6584, 2007.

13. Minner S, Jessen B, Stiedenroth L, et al: Low level HER 2 overexpression is associated with rapid tumor cell proliferation and poor prognosis in prostate cancer. Clin Cancer Res 16: 1553-1560, 2010.

14. Bubendorf L, Sauter G, Moch H, et al: Ki67 labelling index: an independent predictor of progression in prostate cancer treated by radical prostatectomy. J Pathol 178: 437-441, 1996.

15. Erbersdobler A, Isbarn H, Steiner I, et al: Predictive value of prostate-specific antigen expression in prostate cancer: a tissue microarray study. Urology 74: 1169-1173, 2009.

16. El Gammal AT, Bruchmann M, Zustin J, et al: Chromosome 8p deletions and $8 \mathrm{q}$ gains are associated with tumor progression and poor prognosis in prostate cancer. Clin Cancer Res 16: 56-64, 2010.

17. Camp RL, Charette LA and Rimm DL: Validation of tissue microarray technology in breast carcinoma. Lab Invest 80: 1943-1949, 2000.

18. Hoos A, Urist MJ, Stojadinovic A, et al: Validation of tissue microarrays for immunohistochemical profiling of cancer specimens using the example of human fibroblastic tumors. Am J Pathol 158 $1245-1251,2001$.

19. Hoos A, Stojadinovic A, Mastorides S, et al: High Ki-67 proliferative index predicts disease specific survival in patients with high-risk soft tissue sarcomas. Cancer 92: 869-874, 2001.

20. Hoos A, Stojadinovic A, Singh B, et al: Clinical significance of molecular expression profiles of Hurthle cell tumors of the thyroid gland analyzed via tissue microarrays. Am J Pathol 160 175-183, 2002.

21. Engellau J, Akerman M, Anderson $\mathrm{H}$, et al: Tissue microarray technique in soft tissue sarcoma: immunohistochemical Ki-67 expression in malignant fibrous histiocytoma. Appl Immunohistochem Mol Morphol 9: 358-363, 2001.

22. Fernebro E, Dictor M, Bendahl PO, Ferno M and Nilbert M: Evaluation of the tissue microarray technique for immunohistochemical analysis in rectal cancer. Arch Pathol Lab Med 126: 702-705, 2002
23. Zhang D, Salto-Tellez M, Putti TC, Do E and Koay ES: Reliability of tissue microarrays in detecting protein expression and gene amplification in breast cancer. Mod Pathol 16: 79-84, 2003.

24. Kristiansen G, Fritzsche FR, Wassermann K, et al: GOLPH2 protein expression as a novel tissue biomarker for prostate cancer: implications for tissue-based diagnostics. Br J Cancer 99: 939-948, 2008.

25. Rubin MA, Dunn R, Strawderman M and Pienta KJ: Tissue microarray sampling strategy for prostate cancer biomarker analysis. Am J Surg Pathol 26: 312-319, 2002.

26. Bauer JJ, Sesterhenn IA, Mostofi KF, McLeod DG, Srivastava S and Moul JW: p53 nuclear protein expression is an independent prognostic marker in clinically localized prostate cancer patients undergoing radical prostatectomy. Clin Cancer Res 1: 1295-1300, 1995.

27. Bubendorf L, Kononen J, Koivisto P, et al: Survey of gene amplifications during prostate cancer progression by high-throughput fluorescence in situ hybridization on tissue microarrays. Cancer Res 59: 803-806, 1999.

28. Singh SS, Qaqish B, Johnson JL, et al: Sampling strategy for prostate tissue microarrays for $\mathrm{Ki}-67$ and androgen receptor biomarkers. Anal Quant Cytol Histol 26: 194-200, 2004.

29. Zellweger T, Gunther S, Zlobec I, et al: Tumour growth fraction measured by immunohistochemical staining of Ki67 is an independent prognostic factor in preoperative prostate biopsies with small-volume or low-grade prostate cancer. Int J Cancer 124: 2116-2123, 2009.

30. Vesalainen SL, Lipponen PK, Talja MT, Alhava EM and Syrjanen KJ: Proliferating cell nuclear antigen and p53 expression as prognostic factors in T1-2M0 prostatic adenocarcinoma. Int $\mathrm{J}$ Cancer 58: 303-308, 1994.

31. Minner S, Wittmer C, Graefen M, et al: High level PSMA expression is associated with early PSA recurrence in surgically treated prostate cancer. The Prostate 71: 281-288, 2011.

32. Kollermann J, Schlomm T, Bang H, et al: Expression and prognostic relevance of annexin A3 in prostate cancer. Eur Urol 54: 1314-1323, 2008

33. Erbersdobler A, Isbarn H, Dix K, et al: Prognostic value of microvessel density in prostate cancer: a tissue microarray study. World J Urol 28: 687-692, 2010. 